\title{
Mating propensity of Indian Drosophila melanogaster populations with D. simulans: a nonadaptive latitudinal cline
}

\author{
A. DAS, S. MOHANTY†, P. CAPY \& J. R. DAVID $¥ *$ \\ Zoology Department, Utkal University, Bhubaneswar, 751004 India, †Department of Life Sciences, Regional College of \\ Education, Bhubaneswar, 751004 India and $\ddagger$ Laboratoire de Populations, Génétique et Evolution, CNRS, 91198 Gifl \\ Yvette Cedex, France
}

\begin{abstract}
Crosses were investigated between a single strain of Drosophila simulans and 28 natural Indian populations of $D$. melanogaster. In each case, a mass culture and 10 isofemale lines of $D$. melanogaster were studied. Crosses were much easier between $D$. melanogaster females and $D$. simulans males than in the reciprocal case. Also, hybrid offspring were easier to obtain by using $D$. melanogaster flies (either males or females) from isofemale lines than from mass cultures. Finally, the crossability, estimated by progeny production, showed a clear-cut latitudinal cline: hybrids were easier to obtain with $D$. melanogaster populations from higher latitudes. As $D$. simulans does not occur in India, this cline does not reflect an evolutionary interaction between the two sibling species.
\end{abstract}

Keywords: Drosophila melanogaster, Drosophila simulans, geographical variation, interspecific hybrids, isofemale lines.

\section{Introduction}

Since the pioneering studies of Sturtevant (1920), numerous papers have considered the crossability of Drosophila simulans with its cosmopolitan sibling $D$. melanogaster. In no-choice experiments hybrids are produced more easily between $D$. melanogaster females and $D$. simulans males than in the reciprocal way (for a review see Lemeunier et al., 1986). The progeny are fully sterile and generally of a single sex corresponding to that of the $D$. melanogaster parent. In nature, when the two species live in sympatry, interspecific hybrids are often observed, and have been described from Europe (Sperlich, 1962; Mensua \& Perez, 1977; Capy et al., 1987; Kamping \& Van Delden, 1988), from America (Tracey et al., 1973) and Japan (Inoue et al., 1990). These hybrids are always females, indicating that matings occurred between a $D$. melanogaster female and a $D$. simulans male.

The sexual isolation is thus far from complete in natural populations. Under laboratory conditions, genetic variability for hybrid production has been demonstrated in both species at the intra- and interpopulation levels by several investigators (Parsons, 1972; Eoff, 1975, 1977; Watanabe et al., 1977;

*Correspondence.
Carracedo \& Casares, 1985, 1987; Inoue et al., 1990; Izquierdo et al., 1992; Welbergen et al., 1992). Studies on recently collected natural populations remain, however, quite rare.

Drosophila melanogaster and D. simulans are two sibling cosmopolitan species but their geographical distributions are different (David \& Tsacas, 1981; Lachaise et al., 1988). So far as we know, D. melanogaster exists in all places of the world with adequate climatic conditions. Drosophila simulans, on the other hand, is completely absent from large geographical areas providing favourable conditions, and especially from West Africa and Eastern Asia including India, Thailand, Malaysia and China. It is also absent from some oceanic islands such as Martinique and Guadeloupe in the Carribean (David \& Capy, 1983).

According to the classical evolutionary theory (Dobzhansky, 1970; Coyne, 1992) behavioural isolation should be reinforced by natural selection when the two species live in sympatry. Inoue et al. (1990) presented some empirical observations in favour of this theory. As $D$. simulans is not present in India, we expected no reinforcement, so that easier crosses should be obtained with native $D$. melanogaster populations. This hypothesis was analysed by studying the mating propensity of Indian $D$. melanogaster with a single tester stock of $D$. simulans. Surprisingly, our data 
revealed a clear-cut latitudinal cline among these populations. Moreover, hybrid production was always much easier by using inbred flies from isofemale lines than outbred flies from the same mass populations.

\section{Materials and methods}

A wild-type $D$. simulans mass culture was obtained from the curator of stocks, Department of Zoology, Banaras Hindu University, and kindly provided by $\mathrm{Dr}$ B. N. Singh. This culture was used throughout this work as a tester stock. Wild-living $D$. melanogaster were collected from various localities in India, between

Table 1 Percentage of females producing hybrid progeny observed in interspecific crosses between a Drosophila simulans laboratory strain and natural populations of $D$. melanogaster collected at various latitudes

\begin{tabular}{|c|c|c|c|c|c|c|}
\hline \multirow[b]{2}{*}{ Population } & \multirow[b]{2}{*}{ Latitude } & \multirow[b]{2}{*}{$N$} & \multicolumn{2}{|c|}{ Omel. ơsim. } & \multicolumn{2}{|c|}{ Qsim. ömel. } \\
\hline & & & Mass & Lines & Mass & Lines \\
\hline Jammu & 32.60 & 61 & 22.0 & 68 & 1.5 & 4 \\
\hline Shiliguri & 26.67 & 58 & 17.0 & 65 & 0.0 & 5 \\
\hline Guwahati & 26.13 & 100 & 23.5 & 65 & 1.5 & 5 \\
\hline Patna & 25.60 & 59 & 19.0 & 61 & 0.5 & 7 \\
\hline Shillong & 25.50 & 66 & 15.0 & 66 & 3.0 & 8 \\
\hline Ghazipur & 25.49 & 78 & 15.0 & 48 & 1.0 & 5 \\
\hline Ondi & 25.47 & 46 & 15.0 & 51 & 0.0 & 8 \\
\hline Varanasi & 25.33 & 167 & 16.5 & 53 & 0.5 & 14 \\
\hline Ramnagar & 25.27 & 150 & 15.5 & 54 & 2.5 & 9 \\
\hline Jamsoti & 25.07 & 147 & 14.0 & 44 & 1.0 & 9 \\
\hline Lowari & 25.04 & 107 & 13.0 & 48 & 1.5 & 6 \\
\hline Mirzapur & 25.01 & 73 & 12.5 & 41 & 1.0 & 5 \\
\hline Jabalpur & 23.10 & 78 & 11.0 & 46 & 0.5 & 7 \\
\hline Itarsi & 22.50 & 63 & 10.0 & 45 & 0.0 & 5 \\
\hline Midnapur & 22.40 & 61 & 9.5 & 30 & 0.0 & 5 \\
\hline Balasore & 21.50 & 82 & 7.0 & 31 & 0.5 & 4 \\
\hline Nagpur & 21.00 & 53 & 8.5 & 22 & 1.5 & 5 \\
\hline Bhubaneswar & 20.20 & 60 & 8.5 & 26 & 1.0 & 7 \\
\hline Bombay & 19.00 & 58 & 6.0 & 22 & 1.5 & 3 \\
\hline Panaji & 15.50 & 43 & 7.0 & 21 & 0.5 & 1 \\
\hline Tirupati & 13.60 & 54 & 4.5 & 13 & 0.0 & 8 \\
\hline Madras & 13.07 & 41 & 5.5 & 12 & 0.0 & 4 \\
\hline Bangalore & 12.93 & 62 & 5.0 & 13 & 0.5 & 3 \\
\hline Mysore & 12.20 & 104 & 4.5 & 14 & 0.0 & 7 \\
\hline Ernakulam & 10.00 & 56 & 4.0 & 17 & 0.0 & 6 \\
\hline Fort Cochin & 9.74 & 62 & 3.0 & 11 & 0.0 & 4 \\
\hline Trivandrun & 8.53 & 57 & 3.0 & 10 & 0.0 & 5 \\
\hline Kanniya kumari & 8.13 & 55 & 3.5 & 10 & 0.0 & 6 \\
\hline
\end{tabular}

$N$ : number of founder $D$. melanogaster females collected in each locality; Mass and lines: mass culture or isofemale lines, respectively. For each population and each cross, 200 females were studied for mass cultures and 100 for isofemale lines.
1988 and 1991. Altogether, 28 populations were available, spanning a latitudinal range of 8.1 to $32.6^{\circ}$ of north latitude. The list of these populations, latitude of origin and numbers of founder females are given in Table 1.

For each population, a mass culture was established as well as 10 isofemale lines. Virgin females were isolated from the first laboratory generation and distributed in groups of five. These young $D$. melanogaster females (aged 6 hours) were introduced into culture vials containing five $D$, simulans males. After 5 days, the females were isolated each in a fresh vial and then checked for adult offspring production. For each isofemale line, two replicates were carried out, so that 100 females (10 lines) were analysed from each population. With mass cultures, crosses were more difficult to obtain so that 200 females ( 40 sets of five pairs) were used for each population.

The reciprocal cross, between $D$. simulans females and $D$. melanogaster males, was also investigated using the same procedure. Groups of five young $D$. simulans females were placed for 5 days with five $D$. melanogaster males of the first laboratory generation. Then each female was isolated and checked for progeny production. For each population of D. melanogaster, 100 and $200 \mathrm{D}$. simulans females were mated with isofemale lines and mass cultures, respectively. All experiments were made in an air-conditioned room at a temperature of $25 \pm 2^{\circ} \mathrm{C}$.

\section{Results}

Experimental data, i.e. the percentage of females producing hybrid progeny, are given in Table 1 . Mean values and relationships with latitude are shown in Table 2 .

Table 2 Mean percentages of females producing hybrid progeny in crosses between 28 natural populations of Drosophila melanogaster and a single laboratory strain of $D$. simulans

\begin{tabular}{llcc}
\hline Cross & Technique & Mean \pm S.E. & $b$ \\
\hline Omelanogaster & Isofemale lines & $35.96 \pm 3.79$ & $0.0947^{* * *}$ \\
đsimulans & Mass cultures & $10.66 \pm 1.12$ & $0.0892^{* * *}$ \\
& Comparison & $4.53^{* * *}$ & \\
Osimulans & Isofemale lines & $5.89 \pm 0.47$ & 0.0984 \\
Omelanogaster & Mass cultures & $0.71 \pm 0.15$ & $0.0644^{* *}$ \\
& Comparison & $7.45^{* * *}$ & \\
\hline
\end{tabular}

$b$, regression coefficient for female mating success (log transformed for $\$$ melanogaster $\times$ ơ simulans) against latitude.

Student's $t$-tests of significance: ${ }^{* *} P \leqslant 0.01,{ }^{* * *} P \leqslant 0.001$. 
As expected, many more matings producing offspring occurred between $D$. melanogaster females and $D$. simulans males than in the reciprocal cross. Two more interesting observations may be drawn from the data.

First, many more offspring were obtained using the isofemale lines of $D$. melanogaster than the mass cultures. This is highly significant when comparisons are made with a $t$-test (Table 2). A nonparametric test also showed that the difference is highly significant.

The second observation is the positive relationship with latitude: mating propensity of $D$. melanogaster increases with latitude of origin of the population. The relationship is more pronounced in crosses between $D$. melanogaster females and $D$. simulans males, and the results are shown Fig. 1. Although linear regressions gave good fits, several nonlinear adjustments were tried and the best fits were obtained with exponential relationships (Fig. 1).

For the reciprocal cross $(D$. simulans female $\times D$. melanogaster male) there seems also to be a positive relationship with latitude, but it is significant in one case only (mass cultures, Table 2). This probably results from the low overall success of this cross.

\section{Discussion and conclusions}

In this study, progeny production has been taken as an indication of a successful mating. It remains possible that more matings did occur, but that they were not accompanied by sperm transfer. Also heterospecific sperm storage could be less efficient and vary according to females and populations.

Our data confirm the usual observation that mating success is easier between $D$. melanogaster females and $D$. simulans males than in the reciprocal way (Sturtevant, 1920 and others; for a review see Lemeunier et al., 1986). In some cases, however, easier crosses between $D$. simulans females and $D$. melanogaster males have been reported (Welbergen et al., 1992). The reasons for such discrepancies are not known.

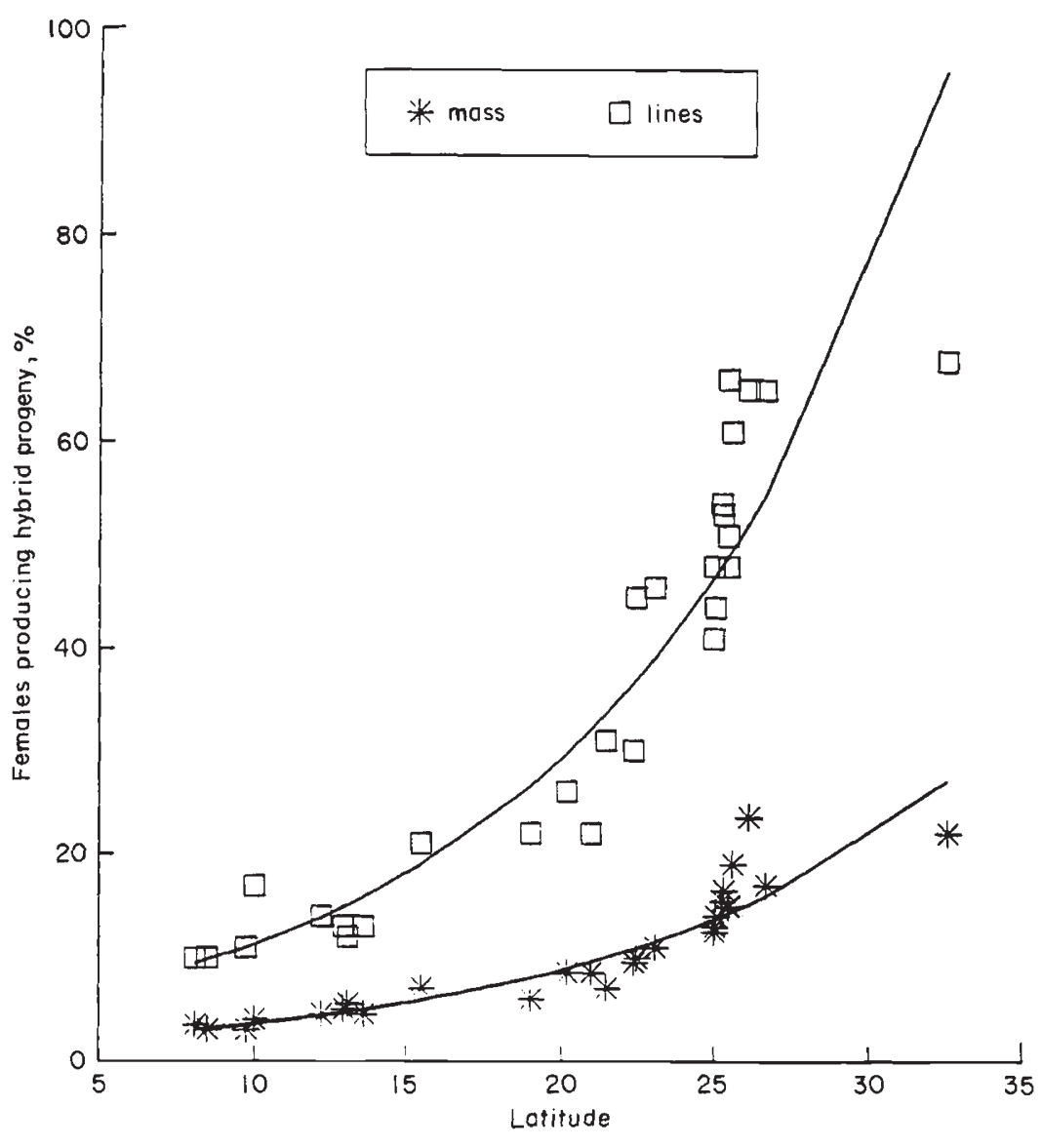

Fig. 1 Relationships between latitude of origin and percentage of Drosophila melanogaster females producing hybrid progeny. Notice that in all cases, insemination was easier with isofemale lines than with mass culture. Transformations were made to exponential regressions, i.e. $y=a e^{b x}$; slopes and tests of significance are in Table 2. 
A clear, although unexpected conclusion of this paper is the better progeny production observed in all cases when using inbred isofemale lines of $D$. melanogaster instead of mass cultures. When $D$. melanogaster females are involved, it could be argued that inbreeding decreases their discriminative capacity as a by-product of an overall lesser fitness. Such an interpretation, however, does not hold for males. A general observation is that inbreeding decreases male sexual activity and mating ability, both in D. melanogaster (Connolly et al., 1974; Welbergen et al., 1992) and D. simulans (Ringo et al., 1977). Under the inbreeding depression hypothesis, we should expect easier matings with inbred females but fewer matings with inbred males. As a better success was observed with inbred flies of both sexes, this interpretation cannot be accepted. We could suggest that the mate recognition system, which maintains homogamy in $D$. melanogaster, is less efficient in inbred lines, but further ethological observations are needed.

The other and novel conclusion concerns the genetic latitudinal cline occurring in Indian natural populations of $D$. melanogaster. As pointed out in the Introduction, numerous investigations have demonstrated that crossability between the two species is a behavioural trait with a strong genetic component, within and between populations. When cultures from various geographical origins are compared, the reported mating frequencies range between 0 and 80 per cent. Such variations demonstrate genetic differences but also the importance of experimental conditions, so that it is almost impossible to compare the data from different authors. Genetic differences may reflect the intrinsic properties of the original populations, but they may also arise from laboratory drift. In the present study, we have two certitudes: first, drift was not involved as experiments were made immediatly after the flies were collected; secondly, mating success did not evolve as an interaction between the two species, as $D$. simulans is absent from India.

In a recent paper, Inoue et al. (1990) compared various populations of $D$. melanogaster from Japan, New Guinea, Africa and Madagascar, and argued that matings were easier with nonsympatric or recently sympatric populations than with ancient sympatric populations, thus favouring the idea of an isolation by reinforcement. We should remember, however, that in countries around the Mediterranean sea, hybrid females have sometimes been found in nature at frequencies of more than 1 per cent (Sperlich, 1962; Mensua \& Perez, 1977; Capy et al., 1987). The two species presumably have coexisted in these Mediterranean countries for a long time (Lachaise et al., 1988), i.e. centuries or millenia. Isolation by reinforcement, if any, would be a very slow process. Looking again at the data of Inoue et al. (1990), we noticed that their seven investigated populations were collected over a broad range of latitudes. Calculating the regression between the percentage of inseminated females (log transformed) and latitude led to a significant relationship ( $b=0.069, P<0.01)$ in spite of the small number of observations. The clear latitudinal cline seen in India could thus be a general characteristic existing in other parts of the world. As a further check of this hypothesis, we performed experimental crosses between a mass culture of a French $D$. simulans, and two mass cultures of D. melanogaster from France and tropical Africa (Congo). In agreement with the above data, crosses were much easier with the French sympatric $D$. melanogaster (45 per cent inseminated females) than with the Congolese strain ( 4 per cent).

Drosophila melanogaster is remarkable for its broad geographical differentiation, and particularly for the occurrence of latitudinal clines for many traits, including behaviour (Lemeunier et al., 1986, David \& Capy, 1988). Finding latitudinal clines, especially when they exist on different continents, is a strong argument for assuming their adaptive significance (Endler, 1977). The cline observed in Indian D. melanogaster populations for their propensity to mate with their sibling species is certainly not a direct adaptation for coexistence with $D$. simulans. More likely it is a by-product of some other sexual behaviour trait, which remains to be identified, and which exhibits latitudinal variations. Possibly the courtship behaviour of $D$. melanogaster evolved in relation to some environmental parameters but independently of its sibling species, in agreement with Paterson's ideas on the evolution of mate recognition (Paterson, 1993).

\section{Acknowledgements}

This work was undertaken in the Genetics Laboratory, Department of Zoology, Banaras Indu University, while A. D. and S. M. were research fellows. Financial assistance was provided by CAS-UGC of B. H. U. and the CSIR of New Dehli.

\section{References}

CAPY, P., DAVID, J. R., CARTON, Y., PLA, E. AND STOCKEL, J. 1987. Grape breeding Drosophila communities in Southern France: short range variation in ecological and genetical structure of natural populations. Acta Oecol., Oecol. Gener., 8, 435-440.

CARRACEDO, M. C. AND CASARES, P. 1985. Intrapopulation genetic variation in the hybridization between Drosophila melanogaster females and D. simulans males. Experientia, 41, 106-108.

(c) The Genetical Society of Great Britain, Heredity, 74, 562-566. 
CARRACEDO, M. C. AND CASARES, P. 1987. Sexual isolation between Drosophila melanogaster females and $D$. simulans males. I. Relation between homospecific and heterospecific mating success. Génét. Sél. Évol., 19, 21-36.

CONNOLLY, K., BURNET, B., KEARNEY, M. AND EASTWOOD, L. 1974. Mating speed and courtship behaviour of inbred lines of Drosophila melanogaster. Behaviour, 48, 61-74.

COYNE, J. A. 1992. Genetics and speciation. Nature, 355, 511-515.

DAVID, J. R. AND CAPY, P. 1983. Drosophila community in domestic habitats of Martinique island, and some specialized breeding sites of native species. Acta Oecol., Oecol. Gener., 4, 265-270.

DAVID, J. R. AND CAPY, P. 1988. Genetic variation of Drosophila melanogaster natural populations. Trends Genet., 4, 106-111.

DAVID, J. R. AND TSACAS, L. 1981. Cosmopolitan, subcosmopolitan and widespread species: different strategies in the Drosophilid family. C. R. Soc. Biogeo., 57, 11-26.

Dobzhansky, т. 1970. Genetics of the Evolutionary Process. Columbia University Press, New York.

ENDler, J. A. 1977. Geographic Variation, Speciation and Clines. Princeton University Press, Princeton, N.J.

EOFF, M. 1975. Artificial selection in Drosophila melanogaster females for increased and decreased sexual isolation from D. simulans males. Am. Nat., 109, 225-229.

EOFF, M. 1977. Artificial selection in Drosophila simulans males for increased and decreased sexual isolation from D. melanogaster females. Am. Nat., 111, 259-266.

INOUE, Y., WATANABE, T. K. AND WATADA, M. 1990. Natural and laboratory hybridization between Drosophila melanogaster and D. simulans. Jap. J. Genet., 65, 47-51.

IZQUIERDO, J. 1., CARRACEDO, M. C., PINEIRO, R. AND CASARES, P. 1992. Response to selection for increased hybridization between Drosophila melanogaster females and $D$. simulans males. J. Hered., 83, 100-104.

KAMPING, A. AND VAN DELDEN, w. 1988. Hybridization between Drosophila melanogaster and D. simulans in nature. Drosoph. Inf. Serv., 67, 53.
LACHAISE, D., CARIOU, M. L., DAVID, J. R., LEMEUNIER, F., TSACAS, L. AND ASHBURNER, M. 1988. Historical biogeography of the Drosophila melanogaster species subgroup. Evol. Biol., 22, 159-225.

LEMEUNIER, F., DAVID, J. R., TSACAS, L. AND ASBURNER, M. 1986. The melanogaster species group. In: Ashburner, M., Carson, H. L. and Thompson J. N. (eds) Genetics and Biology of Drosophila, vol. 3e, pp. 147-256. Academic Press, London.

MENSUA, J. L. AND PEREZ, M. 1977. Hybridization in nature of Drosophila melanogaster and D. simulans. Drosoph. Inf. Serv., 52, 60 .

PARSONS, P. A. 1972. Variations between strains of Drosophila melanogaster and $D$. simulans in giving offspring in interspecific crosses. Can. J. Genet. Cytol., 14, 77-80.

PATERSON, H. E. H. 1993. Evolution and the Recognition Concept of Species. John Hopkins University Press, Baltimore.

RINGO, J, DOWSE, H. AND LAGASSE, S. 1987. Inbreeding decreases mating propensity and productivity in Drosophila simulans. J. Hered., 78, 271-272.

SPERLICH, D. 1962. Hybrids between Drosophila melanogaster and $D$. simulans in nature. Drosoph. Inf. Serv., 36, 118 .

STURTEVANT, A. H. 1920. Genetic studies on Drosophila simulans. I. Introduction: hybrids with $D$. melanogaster. Genetics, 5, 488-500.

TRACEY, M. L., PAVlOSKY, O. AND GREEN, M. M. 1973. Hybridization of Drosophila melanogaster and D. simulans. Drosoph. Inf. Serv., 50, 77.

WATANABE, T. K., LEE, W. H., INOUE, Y. AND KAWANISHI, M. 1977. Genetic variation of the hybrid crossability between Drosophila melanogaster and D. simulans. Jap. J. Genet., 52, 1-8.

WELBERGEN, P., VAN DIJKEN, F. R., SCHARLOO, W. AND KOHLER, W. 1992. The genetic basis of sexual isolation between Drosophila melanogaster and D. simulans, Evolution, 46, 1385-1398. 